\title{
Influence of Ion Beam Damage by FIB on the RESET Amorphous Volume Observation in Phase Change Random Access Memory Device
}

\author{
Jangwon Oh, Youngwoon Jang, Seungjoon Jeon, Taekwon Lee, Won Kim, Ho Joung Kim and Chang \\ Reol Kim
}

Research and Development Division, SKHynix Semiconductor Inc., San 136-1, Ami-ri, Bubal-eub, Ichon-si, Kyoungki-do 467-701, Korea.

Phase change random access memory (PCRAM) device has become the most attractive solution for future memory applications. It is a strong candidate for universal memory because of non-volatile, high speed and high density characteristics. In PCRAM device, data is stored using the difference in electrical resistivity between amorphous (RESET state "1") and crystalline (SET state “0”) phase.

The characterization of amorphous volume after RESET switching in specific cell area is very important for device performance and process developments. Usually, the Transmission Electron Microscope (TEM) cross sectional samples prepared by FIB is used to observe the size and shape of amorphous volume which are critical factors to determine device characteristics. Figure 1 shows the normal structure of PCRAM device consists of a GST layer which is sandwiched between two electrodes, and the mushroom-shape amorphous volume meaning the RESET state. However, sometimes, the amorphous volume was not observed at RESET state. At this time, the TEM results could not provide the reliable data for matching microstructure with electrical data.

Therefore, in this research, we processed double X-sectional TEM analysis using FIB to clarify the reason why the amorphous volume was not observed at RESET state. [1] Figure 2 shows the TEM images of normal X-sectional sample and double X-sectional sample prepared in the same position after RESET switching. In this figure, we confirmed that we could not observe the amorphous volume in GST even after RESET switching, when the Ga ion beam damage induced phase change from amorphous to crystalline happened during TEM sample preparation. And the assessment of the damage impact was obtained by Monte Carlo simulation at Figure3. As a conclusion, we suggest a mechanism for degradation of amorphous volume during TEM analysis and an optimal sample preparation conditions to observe the GST amorphous volume clearly.

References:

[1] L.M. Gignac et al., Microscopy and microanalysis 16 (2010)

[2] J.B. Park et al., Journal of the electrochemical society 154 (2007) 

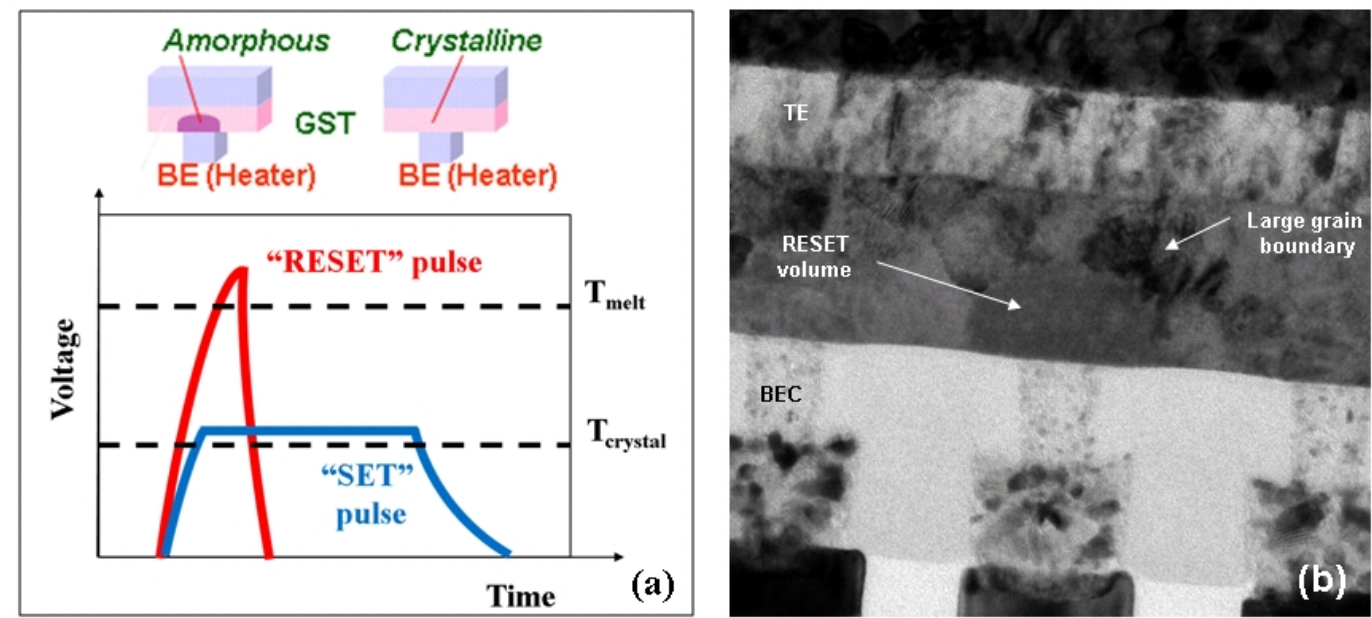

Figure 1. Cross-sectional transmission electron microscopy (TEM) bright field images of a mushroomtype amorphous volume in GST showing RESET state of real cell.

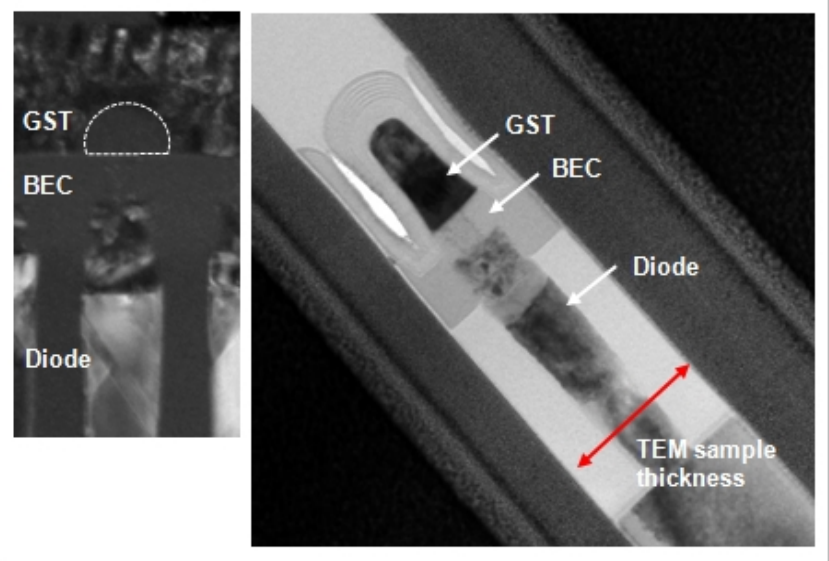

(a) Reset volume (dashed line region) was clearly observed

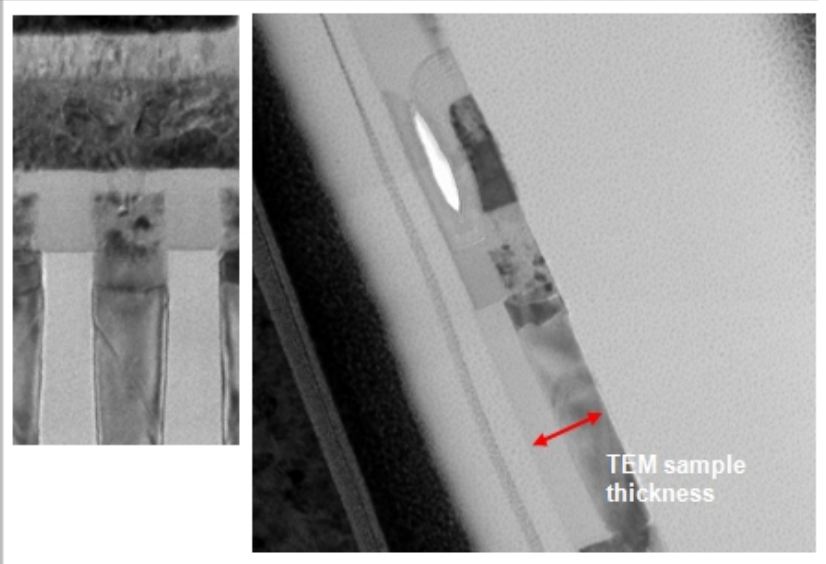

(b) Reset volume was not observed

Figure 2. TEM images of normal cross sectional TEM sample and double cross sectional TEM sample prepared by FIB in the same position.
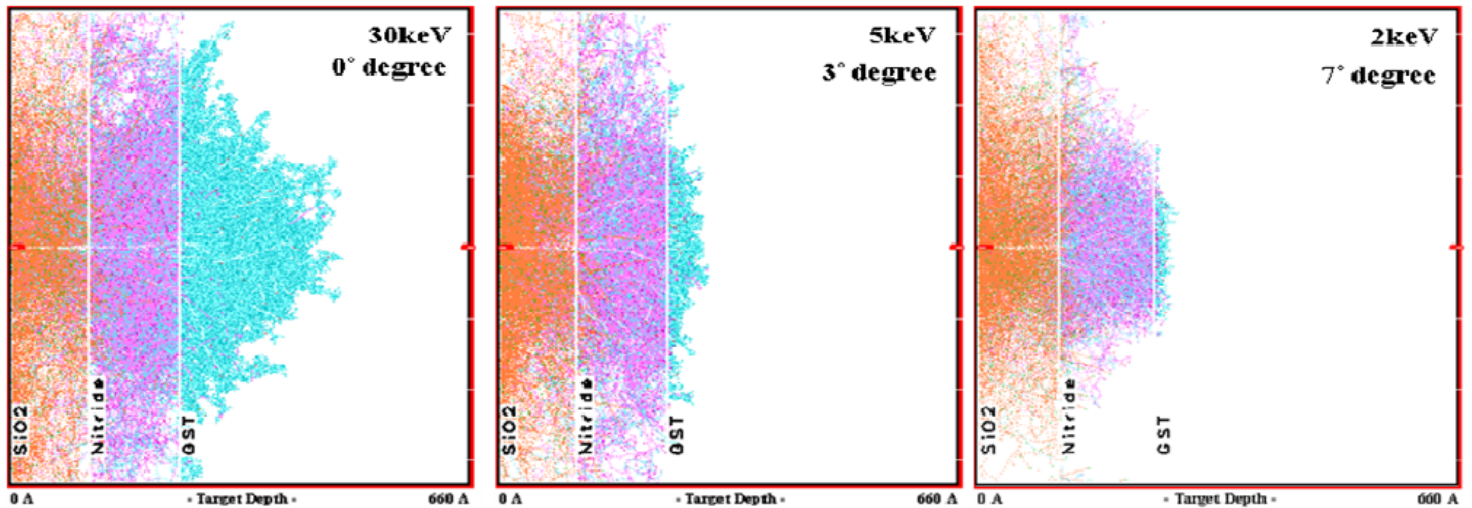

Figure 3. Monte-Carlo simulation results of various acceleration voltage Ga ion condition. Simulations were performed with the SRIM 2008 (Ziegler et al., 2010). The ion beam hits the sample of the FIB sample with an incident angle of $0,3,7$ degrees on the surface from upside down. 